\title{
LÓGICA DEL DESARROLLO ECONÓMICO: BIBLIOTECA UNIVERSITARIA Y CLUSTERS INDUSTRIALES
}

\author{
Alejandro Oyarce-Gatica y Marjorie Mardones
}

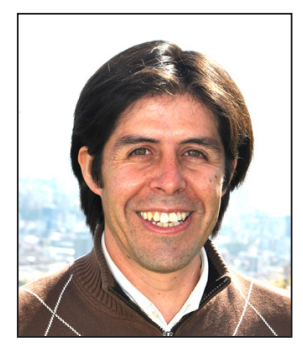

Alejandro Oyarce-Gatica, magister en estudios internacionales por la Universidad de Chile, es bibliotecólogo, licenciado en ciencias de la documentación por la Universidad de Playa Ancha, y diplomado en dirección de servicios por la Universidad Adolfo Ibáñez (UAI). Es coordinador del Departamento de Gestión de la Información y Negocios del Sistema de Bibliotecas de la UAI. Ha sido ponente en congresos internacionales en ciencias de la información en Argentina, Paraguay, Cuba e IFLA. Participa en proyectos de investigación sobre innovación en la calidad de los servicios y alcance de las nuevas tecnologías.

http://orcid.org/0000-0002-2945-1580

Universidad Adolfo Ibáñez. Biblioteca Av. Padre Hurtado 750, 2581793 Viña del Mar, Chile alejandro.oyarce@uai.cl

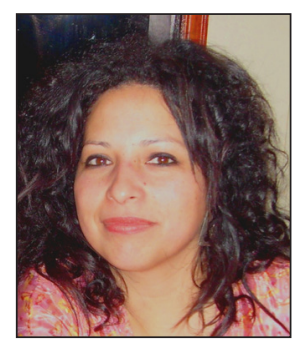

Marjorie Mardones, magister en comunicación local por la Universidad de Playa Ancha (UPLA, Chile), es bibliotecóloga y licenciada en ciencias de la documentación por la UPLA. Coordinadora de la carrera de bibliotecología del Departamento de Ciencias de la Documentación en la Facultad de Ciencias Sociales de la UPLA. Ha destacado por su activa participación en el ámbito cultural, con publicaciones en Uruguay y Chile. Ha sido ponente en encuentros culturales y relacionados con la gestión de bibliotecas, y ha participado en investigaciones con grupos transdisciplinarios, de construcción histórica y patrimonial.

http://orcid.org/0000-0002-0425-1427

Universidad de Playa Ancha Avda. Playa Ancha 850, Casilla 34-V, Valparaíso, Chile marjorie.mardones@upla.cl

\section{Resumen}

En el contexto de la sociedad del conocimiento, es necesario que las bibliotecas universitarias comprendan que sus desafíos están en correlato con la evolución de los tres sectores estratégicos: universidad, Estado e industria. Se analiza la relación entre el desarrollo de la economía y la investigación, y el potenciamiento de los clusters industriales por intermedio de la biblioteca universitaria en el marco de la tercera misión de la universidad.

\section{Palabras clave}

Desarrollo económico, Biblioteca universitaria, Biblioteca académica, Investigación y desarrollo, Clúster industrial, Innovación.

Title: Logic of economic development: university library and industrial clusters

\begin{abstract}
In the context of the knowledge society, it is necessary that university libraries understand that their challenges are correlated with the strategic sectors of national and regional economic development: University, State and Industry. The article seeks to generate an analysis of the relationship between R\&D development and economic development, and of the strategic importance of enhancing industrial clusters through the university library as part of the third mission of universities.
\end{abstract}

\section{Keywords}

Economic development, University library, Academic library, Research and development, Industrial cluster, Innovation.

Oyarce-Gatica, Alejandro; Mardones, Marjorie. "Lógica del desarrollo económico: biblioteca universitaria y clusters industriales". El profesional de la información, 2012, noviembre-diciembre, v. 21, n. 6, pp. 621-626.

http://dx.doi.org/10.3145/epi.2012.nov.09 


\section{Introducción: lógica del desarrollo económico}

Desde siempre, poder y conocimiento han estado estrechamente ligados, y ello es más evidente para los Estados en el marco actual de la sociedad del conocimiento. En palabras de Bell, el poder no surge de la propiedad o de la administración política, sino del conocimiento (Bell, 1976).

A partir de los años 70, gran parte de la inversión privada comenzó a trasladarse desde los sectores industriales a sectores de servicios vinculados con el conocimiento. Drucker, uno de los padres del management empresarial, señalaba que las empresas relacionadas con el conocimiento corresponderían en un futuro no muy lejano a cerca del $50 \%$ del $\mathrm{PIB}$, es decir, que constituirían una parte importante del motor económico de Estados Unidos.

En EUA se creó una vasta red mundial de empresas de servicios relacionadas con la industria del transporte, turismo, y empresas financieras ligadas con la banca y la inversión, entre otras. Este fenómeno se transformó en una tendencia presente en todo el mundo. Diversas instituciones supeditadas a la administración comenzaron a preocuparse por esta situación y durante la "Ronda de Uruguay" en 1993, los Estados partícipes acordaron el nacimiento de la $O M C^{1}$ y la regulación del sector servicios. Con el paso del tiempo, la generación de conocimiento se consolidó como una prioridad. Instituciones de cooperación como la OCDE recomendaron inversiones cuantiosas por parte de los Estados en las áreas de investigación y desarrollo (I+D), enfatizando que la inversión en esta área daría como resultado más y mejor desarrollo de la actividad económica de un país.

\section{Investigación y desarrollo (I+D)}

Es difícil medir el impacto de la I+D en la economía. En 1963 surgió un documento clave para medir la inversión y los esfuerzos por parte de los Estados Ilamado Propuesta de norma práctica para encuestas de investigación y desarroIlo experimental" elaborado por la OCDE. Conocido como Manual de Frascati ${ }^{2}$, su objetivo es establecer definiciones básicas, creando un lenguaje estándar para la comunidad científica, y determinando qué sería considerado investigación y desarrollo (I+D). En el transcurso de los años "las estadísticas sobre estos datos de entrada han demostrado que son indicadores útiles y han sido empleados en informes nacionales e internacionales" (OCDE, 2002). De este modo "las administraciones, interesadas en el crecimiento económico y en la productividad, confían en las estadísticas de I+D como una forma de indicador del cambio tecnológico. Los asesores interesados en la política científica y también en la política industrial, e incluso en las políticas económicas y sociales de carácter general, las utilizan ampliamente. Tales estadísticas constituyen asimismo un punto de partida esencial para numerosos programas gubernamentales, al tiempo que son un instrumento importante para su evaluación" (OCDE, 2002).

Durante las últimas décadas, los países comenzaron a medir su inversión en I+D, monitorizando atentamente la relación entre las inversiones y el crecimiento económico del país. I+D es "el trabajo creativo llevado a cabo de forma sistemática para incrementar el volumen de conocimientos, incluido el conocimiento del hombre, la cultura y la sociedad, y el uso de esos conocimientos para derivar nuevas aplicaciones" (OCDE, 2002).

Existe una etapa no menos importante ligada a la investigación y desarrollo que consiste en la implementación y/o puesta en el mercado de la idea. Esta etapa llamada "innovación" es uno de los temas más abordados y estudiados. Innovación se considera "la implementación de una nueva o significativa mejora de un producto (bien o servicio), proceso, un nuevo método de marketing, un nuevo método organizacional de las prácticas internas del negocio, de la organización del lugar de trabajo o de las relaciones externas" (OCDE, 2009). Han sido muchas las teorías para desarrollar innovación por parte de los Estados, instituciones y empresas privadas con el fin de innovar de la mano de conceptos como "emprendimiento". Schumpeter, economista austríaco de principios de siglo XX, indagó en cómo se producían los cambios tecnológicos en la sociedad (Albornoz, 2009), analizó los procesos de innovación y popularizó términos como la "destrucción creativa", concepto ampliamente utilizado en modelos económicos capitalistas, donde los nuevos productos destruyen antiguas empresas y modelos de negocios (Schumpeter, 1988). Esto se ve reflejado en la introducción de las TICs en los sectores económicos de un país o el paso y convivencia de las industria de bienes tangibles (industria de bienes) a intangibles (industria de servicios).

En las últimas décadas, los países comenzaron a medir su inversión y resultados en I+D, monitorizando la relación entre las inversiones y el crecimiento económico del país

\section{Clusters industriales}

La manera en que los países evolucionaron en busca de la innovación dio lugar a nuevas experiencias estratégicas asociativas. Es así como ciertos capitales industriales se agruparon, dando cabida a lo que hoy se conoce como los clusters industriales. Este concepto fue utilizado por primera vez en 1990 por Porter en su libro Las ventajas competitivas de las naciones. Según este autor "la diversidad e intensidad de las relaciones funcionales entre empresas explican la formación de un complejo productivo y su grado de madurez" (Porter, 1991). Esta idea cambió la manera de analizar los procesos de investigación, el desarrollo y la innovación, y a la vez está ligada con un aspecto territorial, pudiéndose afirmar que: "En lo que la mayoría coincide es que el análisis de clúster es un instrumento analítico de gran utilidad a la hora de describir la complejidad de las actividades productivas para comprender la relación que éstas tienen con el territorio" (Fernández, 2006).

La recomendación de la OCDE en este sentido es crear un sistema nacional de innovación (SIN) con el objetivo de aunar esfuerzos para potenciar los clusters existentes y potenciales, y promoviendo un desarrollo económico focalizado y estratégico con otros países. Canadá, Finlandia, Japón y 
Suiza, entre otros ${ }^{3}$, desarrollaron desde los años 60 sistemas nacionales de innovación (OCDE, 2009), sistematizando esfuerzos para coordinar que diversas instituciones públicas y privadas generaran mayor desarrollo económico. Estos sistemas están gestionados por consejos nacionales de innovación, donde participan el sector industrial, académicos y representantes gubernamentales.

\section{Clusters industriales y universidad}

La idea de la Triple hélice donde universidad, empresa y Estado generan intercambios dinámicos en pos de un crecimiento económico regional (Etzkowitz et al., 2000), es una definición teórico-práctica que busca exponer las fuerzas que convergen en los sistemas nacionales de innovación. Las universidades son los centros generadores por excelencia de I+D y juegan un rol básico en el desarrollo de clusters industriales, basado en la idea de que estos centros de investigación responden a las necesidades generadas por los sectores geográficos industriales en que están insertas: "Los elementos y relaciones que interactúan en la producción, difusión y uso del conocimiento nuevo y útil, desde el punto de vista económico están determinados por una región determinada" (Lundvall, 1992). De este modo, se realizan diversos intentos por facilitar la vinculación entre el sector productivo y la llamada tercera misión de las universidades ${ }^{4}$.

Diversos clusters mundiales potenciados por universidades se han convertido en referentes a seguir. Silicon Valley es uno de estos casos: clúster vinculado a la alta tecnología en ordenadores favorecido por el gobierno de California y donde las universidades cercanas como Berkeley y Stanford participaron activamente en la generación de I+D. Este desarrollo tecnológico desbordó el Valle, impactando en las empresas ubicadas en su entorno. Sin embargo, no es el único caso ${ }^{5}$.

\section{Tercera misión de la universidad}

La biblioteca universitaria debe proporcionar un servicio óptimo y de calidad, capaz de satisfacer los requerimientos de distintos usuarios. Con este fin, debe considerar necesariamente los clusters industriales de la zona donde está ubicada, conociendo e involucrándose en las distintas recomendaciones provenientes del SIN.

En este proceso la biblioteca debe considerar a los stakeholders (interesados) tanto internos como externos, pensando en las nuevas mejoras que debe realizar en la organización, el servicio a sus usuarios y las relaciones con distintos grupos de interés como el sector privado y el Estado, entre otros.

También el personal de la biblioteca universitaria debe valorar su capacidad de intervenir en el entorno participando activamente en los avances sociales, comprendiendo las estrategias de desarrollo propuestas por agentes internacionales, sectores económicos de carácter privado y la gestión del desarrollo proveniente del sector estatal. Así, la sociedad en su conjunto participará en la construcción efectiva de la sociedad del conocimiento, propiciando la mejora en la calidad de vida de quienes la componen.

Respecto al rol del profesional de la información, Rendón señala que: "el profesional de la información es un colega

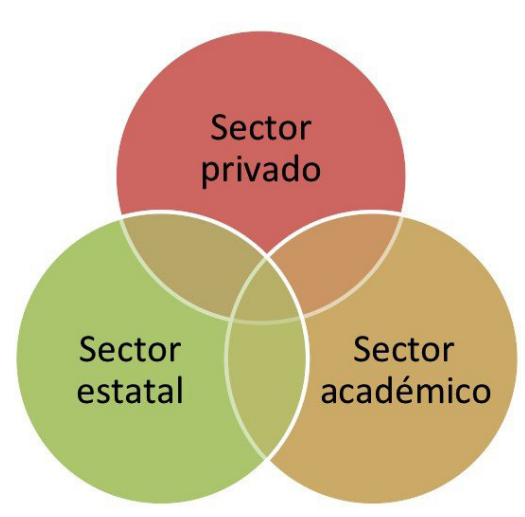

Figura 1. Modelo de la Triple hélice: relación universidad, industria y Estado

dentro de la construcción del conocimiento científico. Como es sabido, una de las características propias del conocimiento científico es que los resultados obtenidos se hacen del dominio de la comunidad científica. Los profesionales de la información, sin ser miembros de la comunidad epistémica de la ciencia particular en que laboran, participan en la vida de ella porque coadyuvan a su existencia, con su actividad permiten que los científicos puedan disponer de una de las herramientas indispensables para su trabajo: la información que los conduce al conocimiento de otros sujetos y a elaborar el suyo propio" (Rendón, 2005).

\section{Clusters y biblioteca universitaria: análisis y propuestas}

El potenciamiento de clusters industriales por parte de los Estados, y su vinculación con las universidades es un fenómeno reciente (el concepto de clúster fue acuñado por Porter en 1990). A pesar de ello, la biblioteca ha realizado esfuerzos por satisfacer las demandas de los investigadores. Pero ¿ participan las bibliotecas de manera consciente en los procesos de desarrollo económico nacionales o regionales? Para optimizar el servicio de las bibliotecas universitarias es necesario que éstas tomen conciencia del fenómeno donde investigación, innovación, clusters y avance económico de una región o un país se interrelacionan, lo que ampliaría considerablemente el rol y alto impacto que tendría la biblioteca universitaria en la construcción de la sociedad del conocimiento.

Para lograr que la biblioteca se centre en las necesidades de los investigadores de I+D referente a los clusters regionales, es necesario que los departamentos rectores de las políticas a investigar en I+D de una universidad tengan la capacidad de valorar la importancia de contar con los recursos informacionales adecuados. El sistema de la biblioteca universitaria debe acoplarse en el proceso de generación de conocimiento de toda la organización en la que está inserta. Los esfuerzos realizados por diversas bibliotecas universitarias a nivel mundial buscando transformarse en crai (centro de recursos para el aprendizaje e investigación) constituyen un paso importante.

Es importante que la organización reconozca cuáles son los clusters industriales ubicados en su radio geográfico. Esta 
información puede ser conseguida por medio de las páginas oficiales de los consejos nacionales o regionales de innovación. Será fácil conocer, por departamentos específicos de las universidades, cuáles son las investigaciones que se realizan. Si se desea buscar "correlación" entre los clusters cercanos y las investigaciones de la universidad se pueden utilizar bases de datos que evalúan el factor de impacto de las publicaciones académicas, como ISI o Scopus ${ }^{6}$.

Las líneas de investigación académica de los docentes e investigadores de la universidad deben ser conocidos por la biblioteca. Estas líneas suelen están en concordancia con los gobiernos centrales o regionales ${ }^{7}$ que buscan potenciar ciertos clusters en el marco de la tercera misión de la universidad. Conocer estas líneas de investigación es el primer paso para integrarlo en el plan estratégico de la biblioteca buscando potenciar la investigación, acompañando y adelantándonos a usuarios con necesidades informacionales específicas.

\section{Las universidades son los centros gene- radores por excelencia de $I+D$}

No existen investigadores de excelencia si no se tiene acceso a recursos bibliográficos de excelencia. Las bases de datos de alto factor de impacto resultan imprescindibles para que los investigadores realicen su tarea. Efectivamente, en diversos países e instituciones ya es tema resuelto, pero en otras aun es un desafío que desacelera el desarrollo del I+D. Algunos de los modelos utilizados por las universidades para resolver esto son: redes de colaboración, acceso a bases de datos a bajo coste o gratis vía Naciones Unidas, acceso financiado directamente por los gobiernos y acceso a publicaciones liberadas de pago ${ }^{8}$.

Los recursos electrónicos y bases de datos deberían enfocarse de forma intuitiva hacia las necesidades de los usuarios, principalmente investigadores que requieren información de publicaciones y patentes referidas a las industrias que buscan potenciar. Esto también abriría espacios para establecer canales de comunicación más estrechos con proveedores de información afines.

El personal de la biblioteca es primordial para implementar nuevos enfoques de servicio y elaborar las estrategias adecuadas para potenciar el rol de la biblioteca bajo esta nueva mirada. Un aspecto destacable es el servicio que debieran realizar los referencistas, pues en la medida que se especialicen en ciertos temas de investigación, podrán orientar mejor a los usuarios, adelantándose a las nuevas demandas y entregando respuestas a las más diversas inquietudes: la referencia virtual y física propia de una biblioteca híbrida viene por añadidura. Ahora bien, las áreas específicas de investigación no sólo deben ser potenciadas con especialistas en bibliometría que presten asesoría a los investigadores sobre las revistas, citas y factores de impacto de las publicaciones; sino también que brinden orientación respecto a patentes, propiedad intelectual e industrial.

\section{Indicadores de medición}

Según lo planteado por Kostoff (1997) el impacto en I+D puede ser medido a partir de tres preguntas clave:

“1) ¿Cuál ha sido la amplitud de los impactos a largo plazo de investigaciones realizadas en el pasado?

2) ¿Cuáles han sido el éxito y los impactos de investigaciones realizadas recientemente?

3) ¿Cuál es el conocimiento que se proyecta ganar de la investigación propuesta, qué tipo de beneficios se podrían obtener y cuál es la probabilidad de que estos resultados a largo plazo puedan ser obtenidos?"

De ello se desprende que la evaluación de los indicadores de impacto de las políticas aplicadas se divide en tres áreas principales:

- Indicadores de impacto en el conocimiento;

- Indicadores de impacto económico;

- Indicadores de impacto social.

\section{Indicadores de impacto en el conocimiento}

Kostoff (1997), relacionando conocimiento e investigación, sostiene que: "Medir el impacto de la investigación requiere la medición de conocimiento. Sin embargo, el conocimiento no puede ser medido directamente. Lo que puede ser observado y medido son las expresiones del conocimiento, como papers, patentes y estudiantes informados. Medidas de expresiones del conocimiento resultantes de la investigación deben, por necesidad, proveer una imagen incompleta del producto de la investigación".

El impacto en el conocimiento del desarrollo de I+D se mide en base a estudios bibliométricos basados en la cantidad de citas y de publicaciones científicas con factor de impacto realizadas por los investigadores de la universidad en las áreas del conocimiento que buscan potenciarse. Estos estudios bibliométricos deben también enfocarse al número de patentes alcanzadas por la universidad y las industrias con las que se relaciona.

\section{Indicadores de impacto económico}

La medición del impacto económico está definido por estándares internacionales normalizados que consideran la Balanza de Pagos por Tecnología ${ }^{9}$ establecida por la $O C D E$ en el Manual de Frascati (OCDE, 2002) que mide el impacto y resultados de la inversión en I+D; y posteriormente el Manual de Oslo que mide la innovación tecnológica (OCDE, 2005), el cual constituye un modelo interactivo que mide la ciencia, la tecnología y la innovación en cada etapa del proceso.

\section{Indicadores de impacto social}

No existen normas internacionales para medir la apropiación del conocimiento en el conjunto de la sociedad por lo que muchas veces se mide este factor de impacto como una prolongación del crecimiento económico el cual deriva finalmente en un desarrollo socioeconómico.

\section{Conclusión}

La biblioteca universitaria necesita adaptarse a las necesidades del medio donde está inserta. La implementación de sistemas nacionales de innovación es una oportunidad no sólo para que la universidad juegue el rol necesario en el marco de la tercera misión, sino también para que la biblio- 
teca académica logre involucrarse de manera consciente en el proceso de generación de conocimiento.

Las mejoras en investigación e innovación tienen por finalidad la materialización de un desarrollo efectivamente sustentable, la optimización de recursos naturales y humanos; y a fin de cuentas, la mejora concreta de la calidad de vida de las personas. Al respecto el Banco Mundial en un Informe para el Desarrollo de 1999 afirma que: "El planteamiento del desarrollo desde la perspectiva del conocimiento (...) puede mejorar las condiciones de vida de formas muy diversas, además de elevar los ingresos. (...) El conocimiento nos permite controlar mejor nuestros destinos".

La biblioteca universitaria necesita adaptarse a las necesidades del medio donde está inserta

\section{Notas}

1. En 1947 nace GATT: General Agreement on Tariffs and Trade, sustituido en 1993 por la OMC (Organización Mundial de Comercio).

2. El Manual de Frascati fue elaborado en la ciudad de Frascati (Italia) por científicos que buscaban normalizar las definiciones de las actividades que realiza el personal de investigación, estándares, recursos invertidos en I+D en sectores públicos y privados del Estado, etc.

3. Los consejos nacionales de innovación fueron creados por diversos países de la OCDE. Entre ellos destacan: Science and Technology Policy Council de Finlandia (1963); Council for Science and Technology del Reino Unido (1993); Austrian Council (2000), Science and Technology Council de Suiza (2000); Council for Science and Technology Policy de Japón (2003); Irish Advisory Council for Science Technology and Innovation (2005); Science, Technology and Innovation Council de Canadá (2007).

4. Se entiende como tercera misión de la Universidad su vinculación con el medio que la rodea, como institución que apoya a la región donde se inserta. Las otras dos misiones son la educación y la investigación.

5. Otros ejemplos: Toulouse (Francia) y la industria aeroespacial, uno de los dos sitios en Europa donde ensamblan los Airbus habiendo establecido acuerdos con diversas universidades entre las que destaca la National Higher School of Aeronautics and Space, considerada una de las mejores de Europa en el área; los de Kista (Suecia) y la industria de tecnologías móviles y computacionales, sede de Ericsson, entre otras empresas vinculadas con el Royal Institute of Technology y la Universidad de Estocolmo; Bengalore (India) y la industria de la subcontratación de software relacionada principalmente con el Indian Institute of Science.

6. La relación entre los clústers industriales cercanos geográficamente y las universidades se puede establecer a través de las bases de datos de ISI o Scopus al analizar cuáles son las áreas de investigación más recurrentes de una universidad en particular y reconocer si investiga sobre temas relacionados con los clusters. Este ejercicio también puede llevarse a cabo observando el índice de patentes industriales aprobadas.

7. Etzkowitz, en la investigación titulada "The future of the university and the university of the future: evolution of ivory tower to entrepreneurial paradigm" analiza la tendencia de las líneas de investigación de las universidades vinculándolas con el medio social e industrial en donde se ubican. Katz también analiza la vinculación de las universidades en EUA con las industrias y la resistencia de las universidades europeas a condicionar la investigación a las necesidades del mercado.

8. Diversas iniciativas públicas y privadas en el marco de los Objetivos del Milenio de la ONU y la responsabilidad social empresarial, se han desarrollado para que los distintos países a nivel global para pueden acceder a las bases de datos de revistas de alto factor de impacto. Estas iniciativas nacen de la Organización Mundial para la Propiedad Intelectual $(O M P I)$ y abarca distintas iniciativas para el acceso a las publicaciones de punta: Hinari, que en conjunto a la OMS se accede a las revistas biomédicas; Agora: que en conjunto con la $F A O$ se accede a publicaciones en el ámbito de la alimentación, agricultura y relativas a las ciencias sociales; y finalmente $O A R E$, programa auspiciado por la Pnuma y la Yale University para que puedan acceder a información relacionada con el medioambiente.

9. La balanza de pagos en tecnología registra las transacciones comerciales relacionadas con la transferencia del conocimiento tecnológico entre un país y el resto del mundo. Esta subcuenta de la balanza de pagos contiene exclusivamente los ingresos y gastos de divisas por el comercio de derechos de uso de tecnologías registradas como propiedad industrial y la prestación de servicios con algún contenido técnico.

\section{Bibliografía}

Albornoz, Mario. "Indicadores de innovación: las dificultades de un concepto en evolución". Revista iberoamericana de ciencia, tecnología y sociedad, 2009, v. 5, n. 13, Buenos Aires.

http://www.scielo.org.ar/scielo.php?script=sci_arttext\&pid $=$ S1850-00132009000200002

Banco Mundial, OCDE. La educación superior en Chile: revisión de políticas nacionales de educación, 2009.

http://mt.educarchile.cl/MT/jjbrunner/archives/La Educación Superior en Chile0407.pdf

Banco Mundial. Informe sobre el desarrollo mundial: el conocimiento al servicio del desarrollo, 1999. ISBN: 08213 $4109 \mathrm{x}$

http://www.ite.educacion.es/formacion/materiales/125/ cd/documentacion_complementaria/9_resumen_informe_ banco_mundial_98_99.pdf

Bell, Daniel. Advenimiento de la sociedad post-industrial: un intento de prognosis social. Madrid: Alianza Editorial, 1976. ISBN: 8420621498

Bitran, Eduardo. "Crecimiento e innovación en Chile". Perspectivas, 2002, v. 5, n. 2, pp. 249-274. 
http://www.inacap.cl/tportal/portales/tp247c7bff9a174/ uploadImg/File/Crecimiento_e_Innovacion_en_Chile_ Fund_Chile.pdf

Castells, Manuel. La era de la información: economía, sociedad y cultura. México: Siglo Veintiuno. 1999, ISBN: 9682321670

Crespo, Manuel; Dridi, Houssine. "Intensification of university-industry relationships and its impact on academic research". Higher education, 2007, v. 54, n. 1, pp. 61-84. http://dx.doi.org/10.1007/s10734-006-9046-0

Dosi, Giovanni; Llerena, Patrick; Sylos Labini, Mauro. "The relationships between science, technologies and their industrial exploitation: an illustration through the myths and realities of the so-called 'European paradox'”. Research policy, 2006, Dec., v. 35, n. 10, pp. 1450-1464.

http://dx.doi.org/10.1016/j.respol.2006.09.012

Ejermo, Olof; Kander, Astrid; Svensson-Henning, Martin. "The R\&D-growth paradox arises in fast-growing sectors". Research policy, 2011, June, v. 40, n. 5, pp. 664-672. http://dx.doi.org/10.1016/j.respol.2011.03.004

Etzkowitz, Henry; Webster, Andrew; Gebhardt, Christiane; Cantisano-Terra, Branca-Regina. "The future of the university and the university of the future: evolution of ivory tower to entrepreneurial paradigm". Research policy, 2000, Febr., v. 29, n. 2, pp. 313-330.

http://dx.doi.org/10.1016/S0048-7333(99)00069-4

Fernández, José-Miguel. Planificación estratégica de ciudades: nuevos instrumentos y procesos. Barcelona: Editorial Reverté, 2006, ISBN: 9788429121100

Garfield, Eugene. "Citation indexes for Science: a new dimension in documentation through association of ideas". Science, 1955, July, v. 122, n. 3159, pp. 108-111. http://dx.doi.org/10.1126/science.122.3159.108

Gobierno de Chile, Consejo Nacional de Innovación para la Competitividad. Agenda de innovación y competitividad 2010-2020.

http://www.cna.gov.co/1741/articles-311056 Agendalnnovacion2010_2020.pdf

Gobierno de Chile, Consejo Nacional de Innovación para la Competitividad. Oportunidades de transformación productiva asociadas a demandas derivadas de los sectores con potencial competitivo (cluster de alto potencial), 2010.

http://biblioteca.cnic.cl/media/users/3/181868/ files/18813/Presentacion_ResumenEjecutivo.pdf

Gobierno de Chile, Ministerio de Economía, Fomento y Turismo, División de Innovación. Resultado de las encuestas de innovación e $1+D$ 2007-2008.

http://www.innventure.cl/wp-content/uploads/2010/08/ innovacion-en-chile.pdf

Katz, Jorge; Contreras, Carmen. "Universidades y desarrollo económico. Nuevos roles de las universidades en la creación y difusión tecnológica: reflexiones sobre el caso chileno". Seminario agenda del desarrollo iberoamericano, educación para el desarrollo en América Latina, 2010, pp. 237-267.

Kostoff, Ronald N. The handbook of research impact as- sessment. Storming Media, 1997, ISBN: 9781423572732

Lundvall, Bengt-Åke. National systems of innovation. Towards a theory of innovation and interactive learning. Pinter Pub. Ltd., 1992. ISBN: 9781855670631

OCDE. Chile's national innovation council for competitiveness. Interim assessment and outlook, 2009.

http://www.cincel.cl/documentos/Recursos/CHILE_ COUNCIL_FINAL.pdf

OCDE. Interim report on the OECD innovation strategy: an agenda for policy action on innovation, 2009.

http://www.oecd.org/site/innovationstrategy/43381127. $p d f$

OCDE. "La innovación tecnológica: definiciones y elementos de base". Redes, 1996, v. 3, n. 6, pp. 131-175.

http://redalyc.uaemex.mx/redalyc/pdf/907/90711287005. $p d f$

OCDE. Manual de Frascati: Propuesta de norma práctica para encuestas de investigación y desarrollo experimental. Fundación Española para la Ciencia y la Tecnología (Fecyt), 2002.

OCDE. Manual de Oslo: Guía para la recogida e interpretación de datos sobre innovación, 2005.

http://www.fia.cl/Portals/O/UPP/Documentos/Manual de Oslo.pdf

Porter, Michael. La ventaja competitiva de las naciones. Buenos Aires: Vergara, 1991. ISBN: 9501511057

Rendón, Miguel-Ángel. Bases teóricas y filosóficas de la bibliotecología. México: Universidad Nacional Autónoma de México, 2005. ISBN: 9703226795

http://librosabiertos.org/bitstream/001/402/8/970-32-2679-5. pdf

Schumpeter, Joseph. Capitalismo, socialismo y democracia. Barcelona: Orbis, 1988. ISBN: 8475302777

Thomas, V. J.; Sharma, Seema; Jain, Sudhir K. "Using patents and publications to assess R\&D efficiency in the states of the USA". World patent information, 2011, March, v. 33, n. 1, pp. 4-10.

http://dx.doi.org/10.1016/j.wpi.2010.01.005

Thorn, Kristian; Soo, Maarja. Latin American universities and the third mission: trends, challenges and policy options. Policy research working paper, World Bank, 2006, August, n. 4002.

http://www-wds.worldbank.org/external/default/ WDSContentServer/IW3P/IB/2006/08/30/000016406_2006 0830142439/Rendered/PDF/wps4002.pdf

Unesco. Hacia las sociedades del conocimiento, 2005. ISBN: 9233040003

http://unesdoc.unesco.org/images/0014/001419/141908s. $p d f$

Vicente-de-Billion, Chloé; Oyarce-Gatica, Alejandro. “¿Está muriendo la biblioteca? Hacia la e-evolución". El profesional de la información, 2010, v. 19, n. 1, pp. 70-76.

http://eprints.rclis.org/handle/10760/14382\#. UJVHBGknC4Y

http://dx.doi.org/10.3145/epi.2010.ene.10 